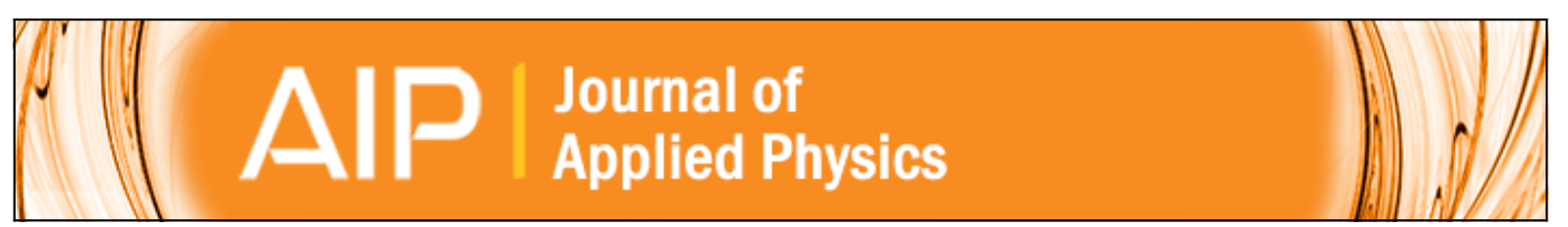

\title{
Effects of coexisting spin disorder and antiferromagnetism on the magnetic behavior of nanostructured (Fe79Mn21)1xCux alloys
}

M. Mizrahi, A. F. Cabrera, S. J. Stewart, and J. Desimoni

Citation: Journal of Applied Physics 115, 213904 (2014); doi: 10.1063/1.4881498

View online: http://dx.doi.org/10.1063/1.4881498

View Table of Contents: http://scitation.aip.org/content/aip/journal/jap/115/21?ver=pdfcov

Published by the AIP Publishing

\section{Articles you may be interested in}

Magnetization jumps and exchange bias induced by a partially disordered antiferromagnetic state in

(FeTiO3)0.9-(Fe2O3)0.1

J. Appl. Phys. 115, 213907 (2014); 10.1063/1.4881527

Observation of spin-glass behavior in antiperovskite compound Mn3Cu0.7Ga0.3N

Appl. Phys. Lett. 103, 022405 (2013); 10.1063/1.4813412

Effect of $\mathrm{Fe}$ to $\mathrm{P}$ concentration ratio on structures, crystallization behavior, and magnetic properties in $(\mathrm{Fe} 0.79+\mathrm{x}$ P0.1 x C0.04B0.04Si0.03)99Cu1 alloys

J. Appl. Phys. 113, 17A337 (2013); 10.1063/1.4800837

Reentrant spin-glass behavior induced by the frustration of Fe-Fe interactions in Laves phase $\mathrm{Nb} 1 \mathrm{x} \mathrm{Hf}$ x Fe2 alloys

J. Appl. Phys. 111, 013913 (2012); 10.1063/1.3674323

Spin glass behavior of mechanically alloyed fcc- ( Fe 79 Mn 21$) 1 \times \mathrm{Cu} \times(0.00 \times 0.30)$

J. Appl. Phys. 107, 044311 (2010); 10.1063/1.3309223

\section{AlP Re-register for Table of Content Alerts}




\title{
Effects of coexisting spin disorder and antiferromagnetism on the magnetic behavior of nanostructured $\left(\mathrm{Fe}_{79} \mathrm{Mn}_{21}\right)_{1-\mathrm{x}} \mathrm{Cu}_{\mathrm{x}}$ alloys
}

\author{
M. Mizrahi, ${ }^{1, \text { a) }}$ A. F. Cabrera, ${ }^{2, a)}$ S. J. Stewart, ${ }^{2,3}$ and J. Desimoni ${ }^{2, b)}$ \\ ${ }^{1}$ INIFTA-CCT- La Plata-CONICET and Departamento de Física, Facultad de Ciencias Exactas, \\ C. C. 67, Universidad Nacional de La Plata, 1900 La Plata, Argentina \\ ${ }^{2}$ IFLP-CCT-La Plata-CONICET and Departamento de Física, Facultad de Ciencias Exactas C.C. 67, \\ Universidad Nacional de La Plata, 1900 La Plata, Argentina \\ ${ }^{3}$ Instituto Ciencias de la Salud, Universidad Nacional Arturo Jauretche, Av. Calchaquí No. 6200, \\ Florencio Varela, Argentina
}

(Received 8 April 2014; accepted 22 May 2014; published online 3 June 2014)

\begin{abstract}
We report a magnetic study on nanostructured $\left(\mathrm{Fe}_{79} \mathrm{Mn}_{21}\right)_{1-\mathrm{x}} \mathrm{Cu}_{\mathrm{x}}(0.00 \leq \mathrm{x} \leq 0.30)$ alloys using static magnetic measurements. The alloys are mainly composed by an antiferromagnetic $f c c$ phase and a disordered region that displays a spin-glass-like behavior. The interplay between the antiferromagnetic and magnetically disordered phases establishes an exchange anisotropy that gives rise to a loop shift at temperatures below the freezing temperature of moments belonging to the disordered region. The loop shift is more noticeable as the $\mathrm{Cu}$ content increases, which also enhances the spin-glass-like features. Further, in the $\mathrm{x}=0.30$ alloy the alignment imposed by applied magnetic fields higher than 4 kOe prevail over the configuration determined by the frustration mechanism that characterizes the spin glass-like phase. (C) 2014 AIP Publishing LLC. [http://dx.doi.org/10.1063/1.4881498]
\end{abstract}

\section{INTRODUCTION}

The magnetic properties of nanoparticles (NPs) are subject of continuously growing interest, driven by both fundamental research and technological applications. Antiferromagnetic (AFM) NPs have received increased attention due to their novel applications as spin valves ${ }^{1}$ and as magnetic random access memory ${ }^{2}$ among others. In these AFM systems, as the particle size diminishes, a net magnetic moment is produced due to the lack of compensation of the magnetic sublattices. In addition, a significant fraction of atoms lies at or near the surface and then surface and interface effects become more and more important. As AFM NPs have a large surface/volume ratio then it is reasonable to correlate their magnetic behavior with surface and finite size effects. Because of the presence of defects, missing bonds, fluctuations in number of atomic neighbors, and inter-atomic distances, the surface shell and the intergrain boundaries are characterized by topological and magnetic disorder. The modification of structural properties near and at the particle surface commonly gives rise to surface anisotropy, weakening of exchange couplings, and surface spin disorder. Furthermore, the structural disorder diverts the surface spin directions from the AFM alignment, affecting the magnetic anisotropy. Therefore, the magnetization is not uniform throughout the particle and the magnetic properties are strongly affected by the nature of the interface exchange coupling between the ordered regions (grain core) and those with a lower degree of ordering (surface layers and/or intergranular space). This coupling can give rise to a shift of the magnetic hysteresis loops after field cooling, i.e., to the exchange-bias (EB) effect. ${ }^{3}$

\footnotetext{
a) Authors to whom correspondence should be addressed. Electronic addresses: mizrahi@fisica.unlp.edu.ar and cabrera@fisica.unlp.edu.ar. ${ }^{\text {b) Deceased. }}$
}

Among AFM systems that present exchange anisotropy when coexisting with other magnetic phases are metal transition oxide nanoparticles, ${ }^{4-6}$ nanocrystalline FeRh alloy ${ }^{7}$ and Mn-based alloys and compounds. ${ }^{3,8}$ Within a core-shell description, mainly used to describe oxide nanoparticle systems, the surface shell behaves as a spin disordered system with spin-glass-like (SG-like) features that couples with the uncompensated AFM core giving rise to the EB phenomenon. ${ }^{4-6,9}$ On the other side, mechanical milling applied to metallic systems produces nanostructured alloys ${ }^{7}$ with ordered regions interconnected by disordered ones. In such cases, an additional complexity could arise when atoms spatially distributed form magnetic clusters that freeze collectively into a superspin glass state. ${ }^{10}$ The exchange anisotropy in Mn-containing alloys has been attributed to the coexistence of AFM and FM interactions due to inhomogeneous distribution of magnetic atoms, or to the intrinsic spinglass behavior in the case of diluted alloys. ${ }^{3,8}$ To provide a better understanding of the magnetism displayed by Mn-based alloys, more investigations are needed on these complex systems when coexisting magnetic phases and/or spin disordered are present.

In previous works, ${ }^{11,12}$ we have reported on the structural, hyperfine and $\mathrm{AC}$-magnetic characterization of nanostructured alloys $\left(\mathrm{Fe}_{79} \mathrm{Mn}_{21}\right)_{1-\mathrm{x}} \mathrm{Cu}_{\mathrm{x}}(0.00 \leq \mathrm{x} \leq 0.30)$ obtained by mechanical milling. These results showed that a ternary $f c c$-FeMnCu random solid solution was formed. Magnetically, the system consists of AFM $f c c$ interacting particles having an intergranular disordered region that confers a SG-like behavior. ${ }^{12}$ Here, we present a more detailed magnetic study on this system, focusing on the role of the disordered region on the magnetic features of these $\left(\mathrm{Fe}_{79} \mathrm{Mn}_{21}\right)_{1-\mathrm{x}} \mathrm{Cu}_{\mathrm{x}}$ alloys. We also explore the EB effect through the measurement of hysteresis loops recorded under different conditions. 


\section{EXPERIMENTAL}

DC magnetic measurements were carried out on $\left(\mathrm{Fe}_{79} \mathrm{Mn}_{21}\right)_{1-\mathrm{x}} \mathrm{Cu}_{\mathrm{x}}$ nanostructured samples (average particle size $\sim 10 \mathrm{~nm}$ ) with nominal compositions $\mathrm{x}=0.00,0.10$, $0.15,0.20,0.25$, and 0.30 . Details of sample preparation and characterization can be found elsewhere. ${ }^{12}$ The thermal dependence of the magnetization were recorded using two different conditions: (i) cooling without applied magnetic field down to $5 \mathrm{~K}$, then a static magnetic field $\left(\mathrm{H}_{\mathrm{DC}}\right)$ was applied during the measurement until reaching $300 \mathrm{~K}$ (Zero Field Cooling-ZFC) and (ii) the cooling process was started in presence of the same magnetic field used in the ZFC measurements keeping the field during the warming process (Field Cooling-FC). These measurements were performed using $\mathrm{H}_{\mathrm{DC}}=20$ and $100 \mathrm{Oe}$, whereas for sample $\mathrm{x}=0.30$ $\mathrm{H}_{\mathrm{DC}}=200,400$, and 4000 Oe were also employed.

$M$ vs. $H$ curves were recorded at several temperatures within the 5-300 K range with applied magnetic fields up to
$50 \mathrm{kOe}$. Low temperature measurements were performed in ZFC condition. Hysteresis loops under FC-conditions were also measured for $\mathrm{x}=0.00,0.20$, and 0.30 samples in the 5 to $300 \mathrm{~K}$ range. In the $\mathrm{FC}$ procedure, the sample was cooled down from $300 \mathrm{~K}$ under applied fields $\left(\mathrm{H}_{\mathrm{DC}}\right)$ between $250 \mathrm{Oe}$ and $2.5 \mathrm{kOe}$. Once the measuring temperature was achieved, the field was set at $\mathrm{H}=0 \mathrm{Oe}$ and the measurement of the loop started. All measurements were performed in a SQUID magnetometer (Lakeshore MPMS XL).

\section{RESULTS AND DISCUSSION}

Figs. 1(a) and 1(b) shows the FC and ZFC magnetization curves registered under fields $\mathrm{H}_{\mathrm{DC}}=20$ and $100 \mathrm{Oe}$, respectively. In all cases, the ZFC curves show a broad peak with a maximum at the temperature $T_{\mathrm{ZFC}}$ (see Table I), which is more pronounced for high $\mathrm{Cu}$ concentrations. This temperature represents the temperature of freezing of moments belonging to the disordered regions. ${ }^{12}$ We observe that $\mathrm{FC}$
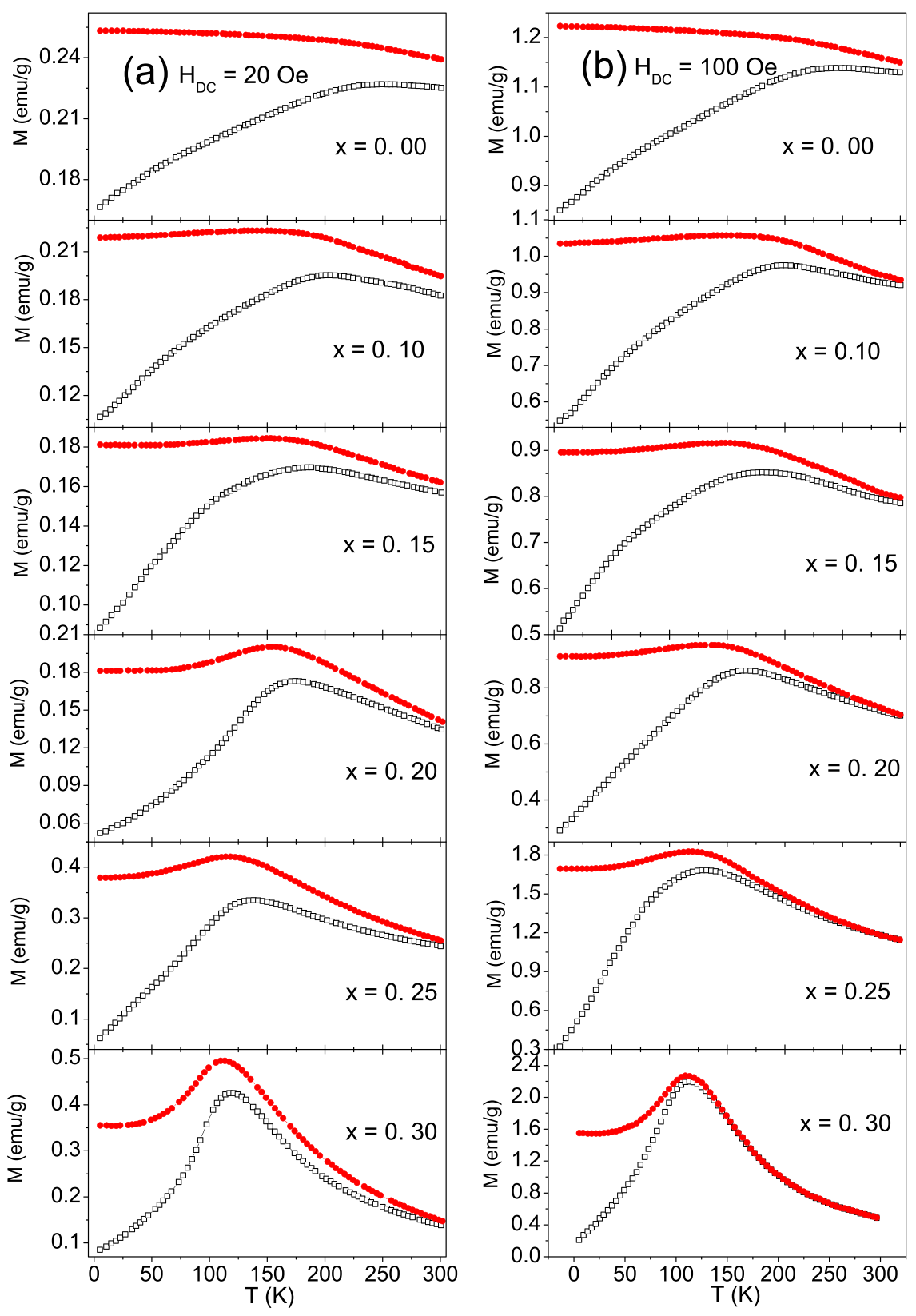

FIG. 1. ZFC (open squares) and FC (solid circles) static magnetization under magnetic applied fields of $20 \mathrm{Oe}$ (a) and $100 \mathrm{Oe}(\mathrm{b})$. 
TABLE I. Characteristic temperatures obtained from ZFC-FC magnetization and susceptibility curves. $T_{\mathrm{i}}$ is the irreversibility temperature, $T_{\mathrm{ZFC}}$ is the temperature where the ZFC maximum occurs, and $\mathrm{T}_{\text {susc }}$ is the temperature where the AC-susceptibility occurs. ${ }^{12} \mathrm{H}_{\mathrm{DC}}$ is the applied static magnetic field.

\begin{tabular}{lcccccc}
\hline \hline $\mathrm{x}$ & 0.00 & 0.10 & 0.15 & 0.20 & 0.25 & 0.30 \\
\hline$T_{\mathrm{i}}(\mathrm{K})\left(\mathrm{H}_{\mathrm{DC}}=20 \mathrm{Oe}\right)$ & $>300$ & $>300$ & $>300$ & $>300$ & 281 & 257 \\
$T_{\mathrm{i}}(\mathrm{K})\left(\mathrm{H}_{\mathrm{DC}}=100 \mathrm{Oe}\right)$ & 224 & 214 & 203 & 192 & 159 & 101 \\
$T_{\mathrm{ZFC}}(\mathrm{K})\left(\mathrm{H}_{\mathrm{DC}}=20 \mathrm{Oe}\right)$ & 246 & 201 & 185 & 171 & 134 & 116 \\
$T_{\mathrm{ZFC}}(\mathrm{K})\left(\mathrm{H}_{\mathrm{DC}}=100 \mathrm{Oe}\right)$ & 241 & 196 & 179 & 163 & 127 & 110 \\
$T_{\text {susc }}(\mathrm{K})\left(\mathrm{H}_{\mathrm{DC}}=0 \mathrm{Oe}\right)$ & 250 & 210 & 195 & 163 & 138 & 120 \\
\hline \hline
\end{tabular}

curves remain approximately constant at temperatures lower than $T_{\mathrm{ZFC}}$, being this behavior usually observed in a spin glass-like system. ${ }^{13}$ A curious trend in the evolution of the maximum of the magnetization with the $\mathrm{Cu}$ content can be observed, decreasing from $\mathrm{x}=0$ until $\mathrm{x}=0.15$ and increasing for $x \geq 0.20$. This behavior can be explained in terms of competition between two magnetic phases present in the samples. I.e. the ferromagnetically ordered bcc- $\mathrm{Fe}(\mathrm{Mn}, \mathrm{Cu})$ that decreases with $\mathrm{x}$ and the SG phase, which begins to be more important at high $\mathrm{Cu}$ content. ${ }^{12}$ The irreversibility temperature $\left(T_{\mathrm{i}}\right)$, which is the temperature where the ZFC magnetization curve departs from the FC one, ${ }^{14} \mathrm{~T}_{\mathrm{ZFC}}$ and the AC susceptibility maximum $\left(T_{\text {susc }}\right)$ temperatures ${ }^{12}$ are shown in Table I.

For all samples $T_{i}$ decreases with the $\mathrm{Cu}$ content (see Figs. 1(a) and 1(b), Table I). Particularly, by applying a field $\mathrm{H}_{\mathrm{DC}}=100 \mathrm{Oe}$, which is higher than the coercive fields registered at $300 \mathrm{~K}$, we observe that the irreversibility starts

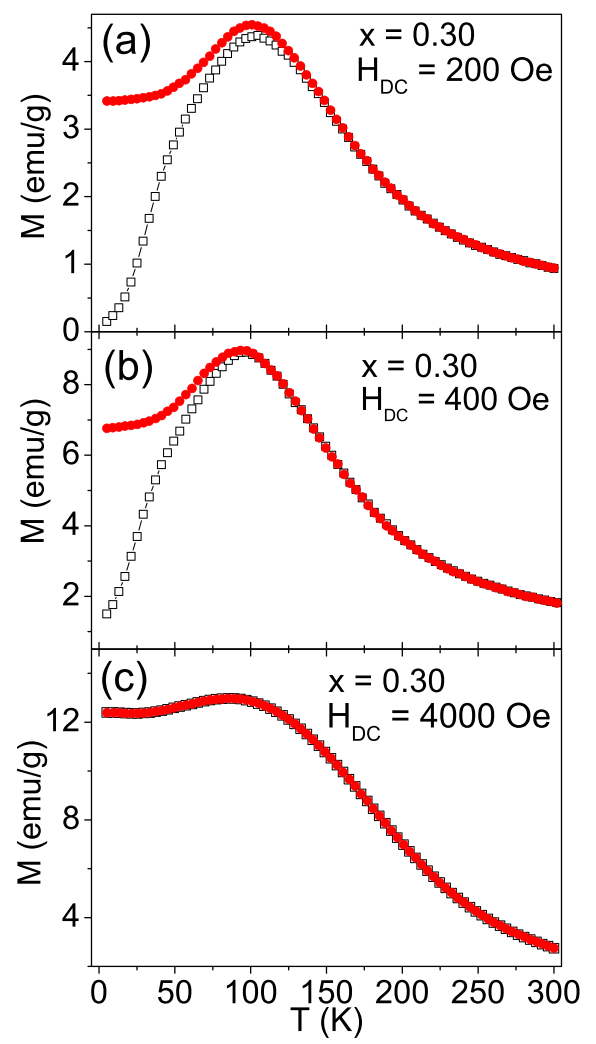

FIG. 2. Thermal dependence of the ZFC-FC magnetization corresponding to $\mathrm{x}=0.30$ sample under fields of 200 (a), 400 (b), and 4000 Oe (c). below room temperature (RT). The presence of a small fraction of ferromagnetically ordered $b c c-\mathrm{Fe}(\mathrm{Mn}, \mathrm{Cu})$, which is detectable for samples $\mathrm{x}<20,{ }^{12}$ contributes to the separation between ZFC and FC curves. This phase is also responsible for the substantial difference between $T_{\mathrm{i}}$ and $T_{\mathrm{ZFC}}$, which is larger than that expected for canonical spin glasses. ${ }^{15}$ Further, $T_{\mathrm{i}}$ and $T_{\mathrm{ZFC}}$ shift to lower values as the amount of $\mathrm{Cu}$ increases, and ZFC-FC curves collapse when $\mathrm{H}_{\mathrm{DC}}=$ $4 \mathrm{kOe}$ for the $\mathrm{x}=0.30$ sample (Fig. 2).

Independently on the $\mathrm{Cu}$ concentration, $T_{\mathrm{ZFC}}$ is lower than $T_{\text {susc }}$. Such difference is originated in the dependence of $T_{\mathrm{ZFC}}$ and $T_{\text {susc }}$ with: (i) the temporal window that characterizes these measurements, ${ }^{12}$ and (ii) the magnitude of the applied DC magnetic fields $\left(\mathrm{H}_{\mathrm{DC}}\right)$. Fig. 3(a) shows the variation of the measuring time $(\tau)$ as function of the inverse of the characteristic temperature $(1 / T)$ for $\mathrm{x}=0.30$, and Fig. 3(b) shows $T_{\mathrm{ZFC}}$ and $T_{\text {susc }}$ as function of $\mathrm{H}_{\mathrm{DC}}$ for the same sample. The characteristic temperature is defined as the temperature for which the relaxation time of the moments of the disordered regions equals to $\tau$. The first graph clearly shows that these data cannot be well reproduced by an Arrhenius type law. ${ }^{16}$ However, the Vogel-Fulcher law ${ }^{17}$ reproduces quite well the behavior indicating the presence of interactions between the moments.
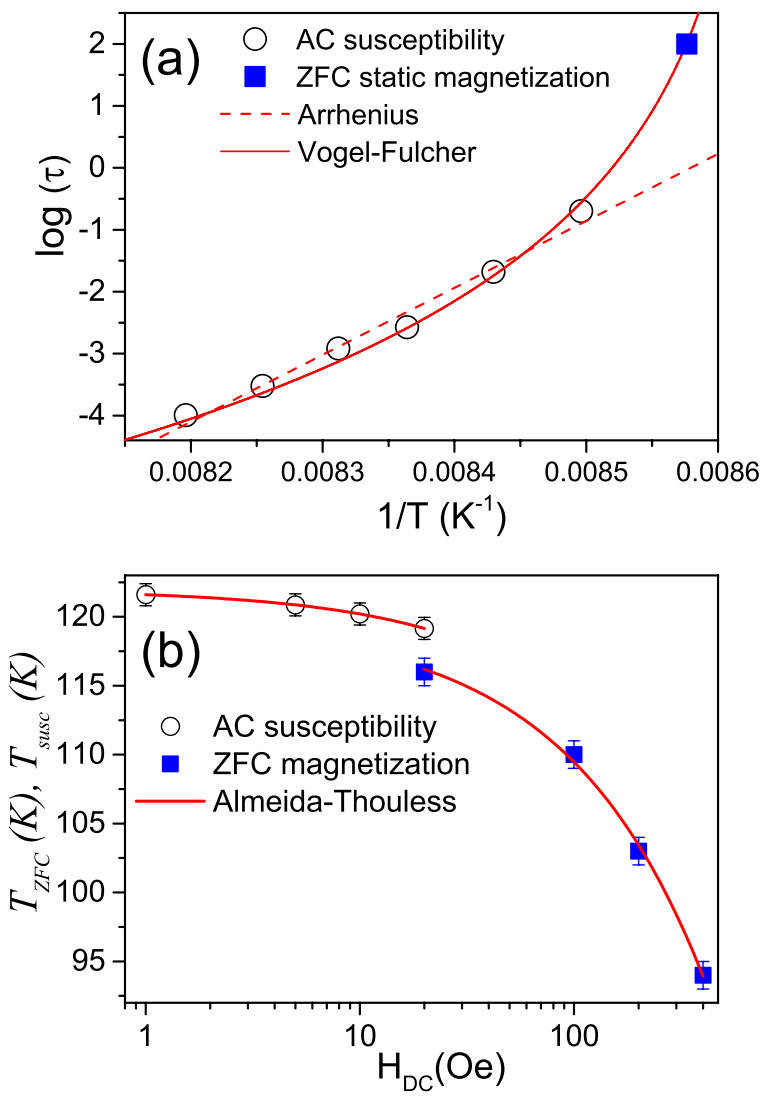

FIG. 3. (a) Relaxation time ( $\tau$ ) from AC susceptibility (open circles) and DC magnetization (solid squares) as a function of the inverse of the characteristic temperature (1/T). Dashed line and solid line are the fits using the Arrhenius's law and the Vogel-Fulcher's law, respectively. (b) Characteristic temperature from AC susceptibility (open circles) and DC magnetization (solid squares) as a function of the DC applied field for $\mathrm{x}=0.30$ sample. 
From here it is possible to extrapolate the characteristic temperature expected for Mössbauer spectroscopy measurements (i.e., when $\tau=10^{-8} \mathrm{~s}$ ), which results about $130 \mathrm{~K}$, in good agreement with previous experimental findings. ${ }^{12}$ The dependence of $T_{\mathrm{ZFC}}$ and $T_{\text {susc }}$ with $H_{D C}$ follow the de Almeida and Thouless relationship: ${ }^{18}$

$$
T=T_{0}-a\left(H_{D C}\right)^{2 / 3}
$$

where $T_{O}$ is a fitting parameter associated to the freezing temperature of the system extrapolated to $H_{D C}=0$ and $a$ is a proportionality factor. The resulting $T_{0}$ values are 122(1) and 119(1) K for AC and DC magnetic measurements results, respectively. Notably these critical temperatures are similar to those reported in Table I. The extrapolation of the curve to $T=0 \mathrm{~K}$ gives a critical cooling field $H_{C F C} \sim 4 \mathrm{kOe}$ from which the spin glass behavior would be negligible. Interestingly, the ZFC-FC curves coincide when $H_{D C}=4 \mathrm{kOe}$ (Fig. 2(c)).
Fig. 4 shows the hysteresis loops for $\mathrm{x}=0.00,0.15$, and 0.30 recorded at different temperatures from ambient down to $5 \mathrm{~K}$. We observe that the general trend of these cycles is similar. At $300 \mathrm{~K}$, the loops show hysteretic behavior and an $\mathrm{S}$-shape. Saturation is not achieved even for fields as high as $50 \mathrm{kOe}$. Both the magnetization $\left(M_{\mathrm{S}}\right)$ measured at $50 \mathrm{kOe}$ and the coercive field $\left(H_{\mathrm{c}}\right)$ decrease with the $\mathrm{Cu}$ content. Both $H_{\mathrm{C}}$ and $M_{\mathrm{S}}$ increase with decreasing temperature. Furthermore, the linear increment of the magnetization at high fields $(H>30 \mathrm{kOe})$ almost does not change with the temperature, being only dependent on the $\mathrm{Cu}$ content. At low temperatures and fields, the loop curvature suggests the presence of another magnetic phase in addition to the $b c c-\mathrm{FeMnCu}$ one. This fact is confirmed by the asymmetry displayed at low temperatures by the field derivative of magnetization $(\mathrm{d} M / \mathrm{d} H)$ shown in Fig. 4 (right), which would correspond by at least two magnetic ordered phases with different coercive fields.
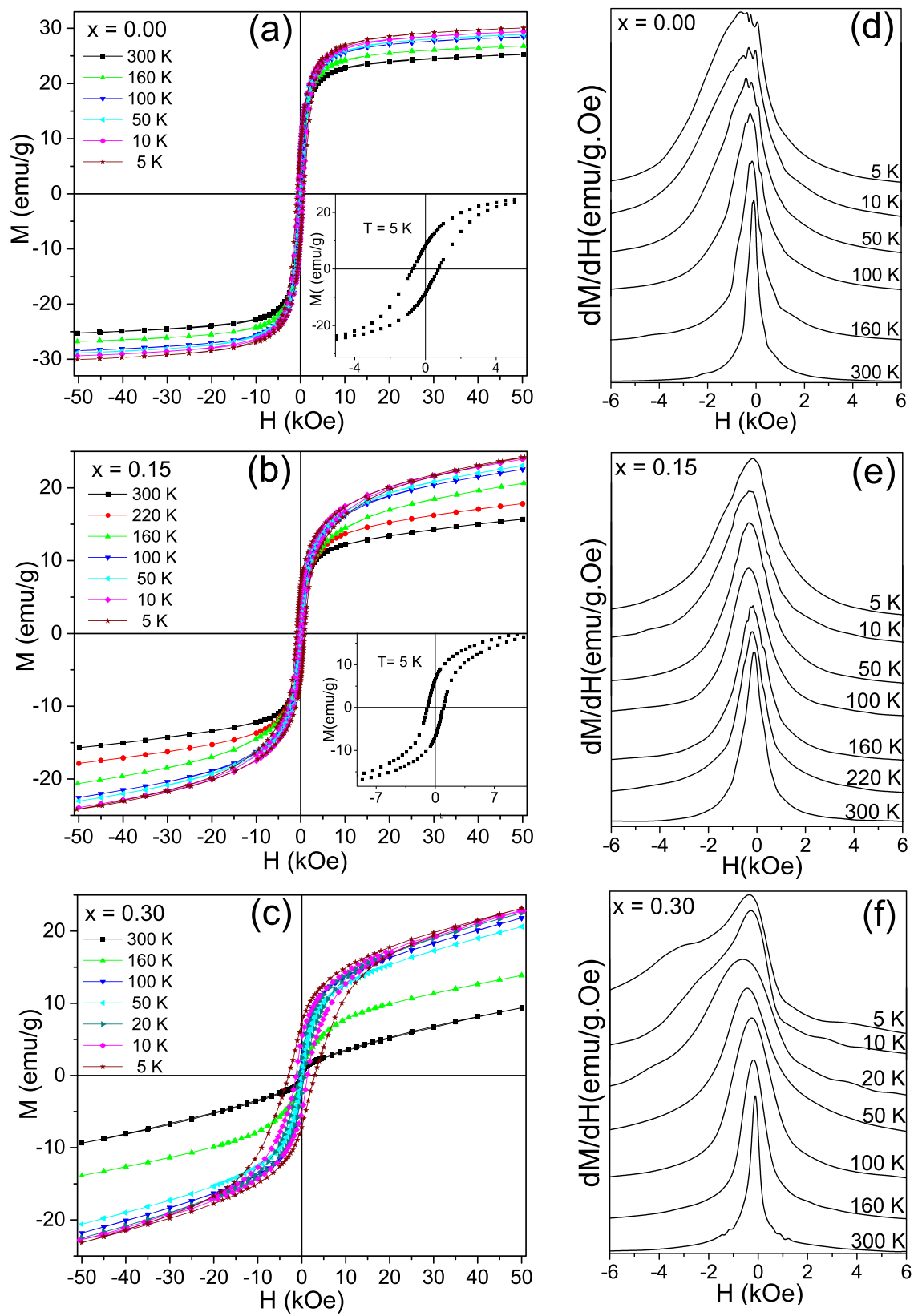

FIG. 4. Left: hysteresis loops taken between $300 \mathrm{~K}$ and $5 \mathrm{~K}$ for samples $\left(\mathrm{Fe}_{79} \mathrm{Mn}_{21}\right)_{1-\mathrm{x}} \mathrm{Cu}_{\mathrm{x}}$ with $\mathrm{x}=0.00$ (a), $x=0.15$ (b), and $x=0.30$ (c). Right: derivative of the magnetization with respect to the applied field of the left branch of the hysteresis loops for $\mathrm{x}=0.00 \quad(\mathrm{~d}), \mathrm{x}=0.15 \quad$ (e), and $\mathrm{x}=0.30$ (f). 

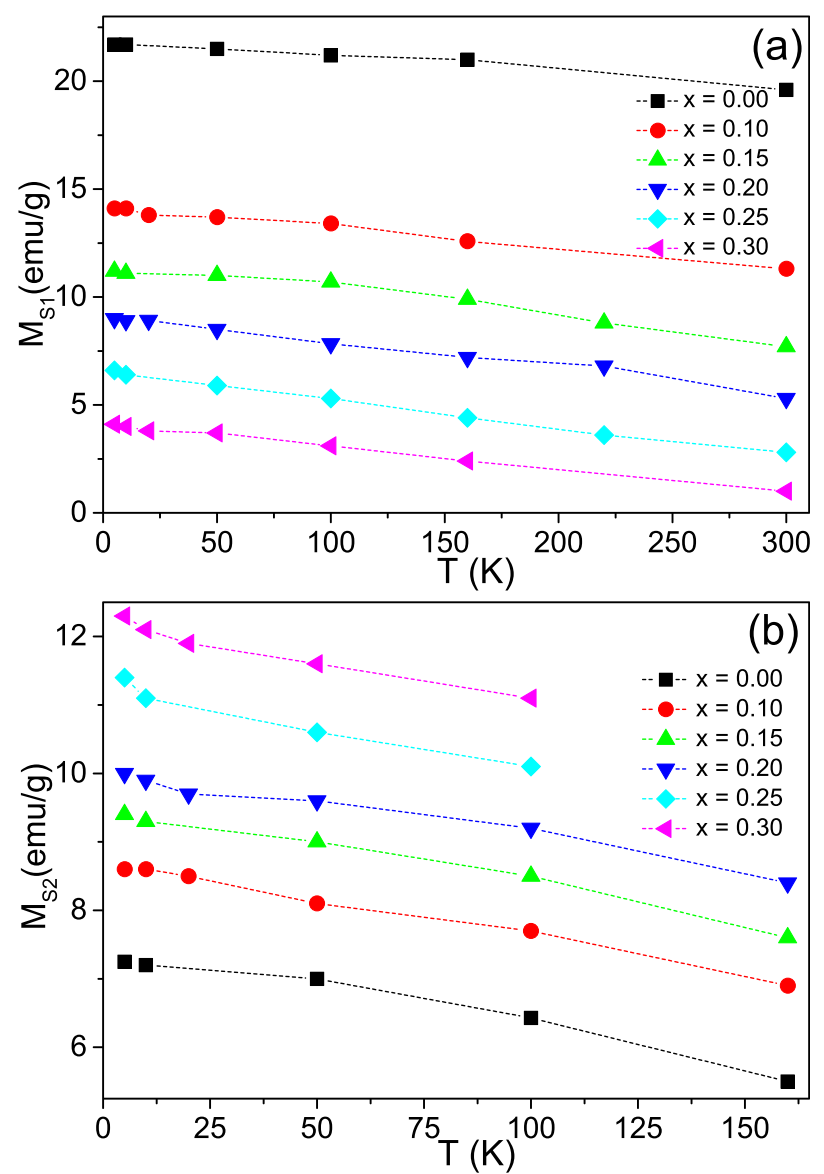

FIG. 5. (a) Saturation magnetizations $\mathrm{M}_{\mathrm{S} 1}$ and (b) $\mathrm{M}_{\mathrm{S} 2}$ of the ferromagnetic components F1 and F2 (see text) as a function of the temperature.

Based on these observations, these cycles were modeled in the following way: $(i)$ for cycles measured at $T>T_{\mathrm{ZFC}}$, we have considered a ferromagnetic contribution (F1, with magnetization $M_{\mathrm{F} 1}$, a Langevin function $\left(M_{L}\right)$ and a linear component $\left(M_{A}\right)$ (see Eq. (2) (Ref. 19)), and, (ii) for cycles registered at $T<T_{\mathrm{ZFC}}$, the Langevin function was replaced by a second ferromagnetic component (F2, with magnetization $M_{F 2}$ ) (see Eq. (3) (Ref. 19)).

$$
\begin{aligned}
M= & M_{F 1}+M_{L}+M_{A} \\
= & M_{S 1}\left[\operatorname{arctg}\left(\frac{\mathrm{H} \pm H_{C 1}}{H_{C 1}} \operatorname{tg}\left(\frac{S_{1}}{\pi}\right)\right)\right] \\
& +M_{S L}\left[\operatorname{cotgh}\left(\frac{\mu \mathrm{H}}{\mathrm{k}_{\mathrm{B}} \mathrm{T}}\right)-\frac{\mathrm{k}_{\mathrm{B}} \mathrm{T}}{\mu \mathrm{H}}\right]+\chi_{A} \mathrm{H}, \\
M= & M_{F 1}+M_{F 2}+M_{A} \\
= & M_{S 1}\left[\operatorname{arctg}\left(\frac{\mathrm{H} \pm H_{C 1}}{H_{C 1}} \operatorname{tg}\left(\frac{S_{1}}{\pi}\right)\right)\right] \\
& +M_{S 2}\left[\operatorname{arctg}\left(\frac{\mathrm{H} \pm H_{C 2}}{H_{C 2}} \operatorname{tg}\left(\frac{\mathrm{S}_{2}}{\pi}\right)\right)\right]+\chi_{A} \mathrm{H},
\end{aligned}
$$

where $M_{S 1}$ and $M_{S 2}$ are the saturation magnetizations, $H_{C 1}$ and $H_{C 2}$ are the coercive fields and $S_{1}$ and $S_{2}$ are the squareness (ratio of the remanent to saturation magnetization) of the ferromagnetic components F1 and F2, respectively. $M_{S L}$ is the maximum magnetization of the Langevin contribution and $\chi_{\mathrm{A}}$ is the paramagnetic susceptibility. Figs. 5 and 6 show the best fitting parameters.

At RT we observe that $M_{S 1}$ decreases from 20 to $1 \mathrm{emu}$ per gram of sample as the $\mathrm{Cu}$ concentration goes from $\mathrm{x}=0.00$ to $\mathrm{x}=0.30$. In all cases, we notice a slight increase of $M_{\mathrm{S} 1}$ as the measurement temperature is lowered (see Fig. 5). These $M_{\mathrm{S} 1}$ values are consistent with those expected considering the fraction of $b c c-\mathrm{FeMnCu}$ phase estimated from Mössbauer spectroscopy results. ${ }^{12}$ The $b c c$ phase fraction is even lower for $\mathrm{x}=0.30$, being below the Mössbauer detection limit. Thus, the F1 component can be ascribed to this secondary minority phase. At $T>T_{\mathrm{ZFC}}$, the disordered phase located at the intergranular regions is represented by the $M_{L}$ component, meaning that as the temperature exceeds the freezing temperature the disordered spin regions are decoupled and present a superparamagnetic-like relaxation. ${ }^{12}$ The F2 magnetic contribution appears for temperatures below $T_{\mathrm{ZFC}}$, and we associate it with the ordering (SG-like state) and/or blocking (super-SG state) of the disordered regions. $M_{S 2}$ increases as the temperature decreases and the rate of change is more pronounced than in $M_{S 1}$. Contrary to $M_{S 1}, M_{S 2}$ increases as $x$ increases at all temperatures. On the other hand, the coercive field parameters, $H_{C 1}$ and $H_{\mathrm{C} 2}$ (Fig. 6), increase as the temperature decreases. It is worth mentioning that the $\chi_{A}$ values remain almost constant for all temperatures $\left(\chi_{A} \approx 10^{-5}-10^{-4} \mathrm{emu} \mathrm{g}^{-1} \mathrm{Oe}^{-1}\right)$. This temperature-independent term probably arises from the
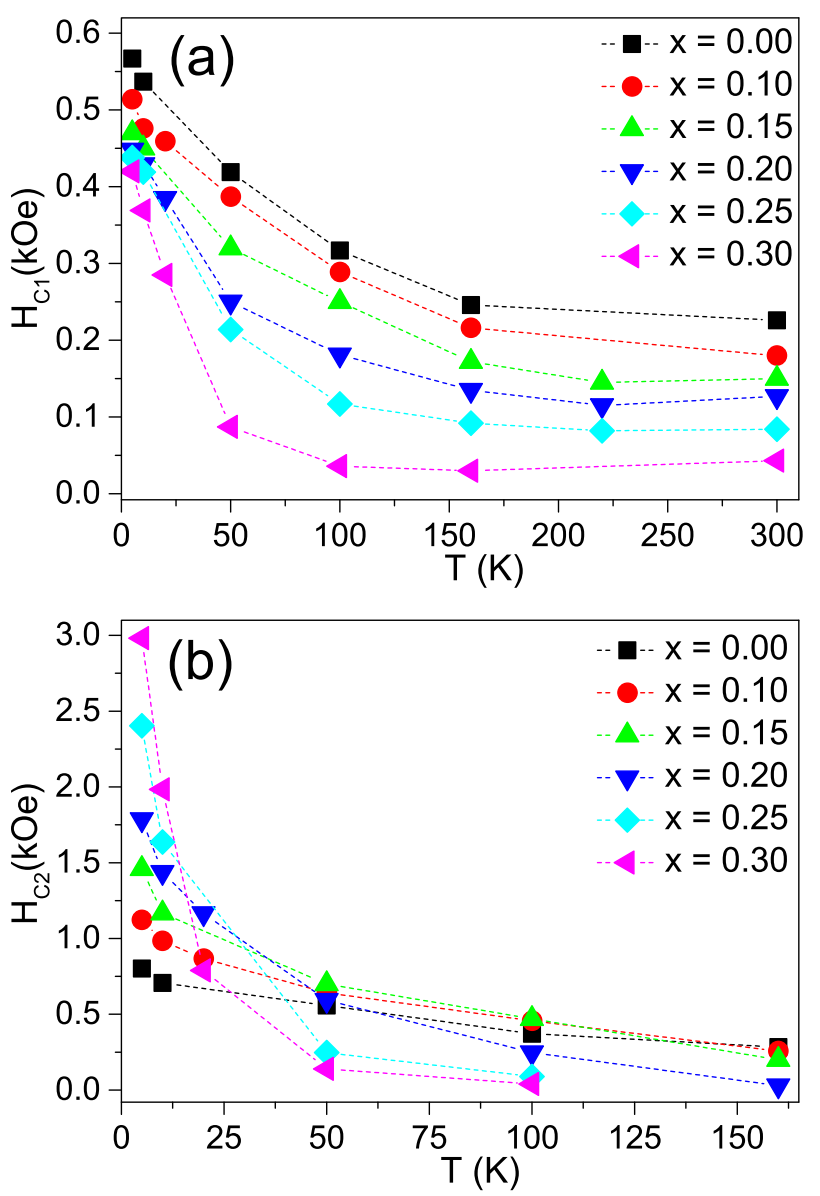

FIG. 6. (a) Coercive field $\mathrm{H}_{\mathrm{C} 1}$ and (b) $\mathrm{H}_{\mathrm{C} 2}$ dependences with the temperature, of $\mathrm{F} 1$ and $\mathrm{F} 2$ ferromagnetic contributions. 


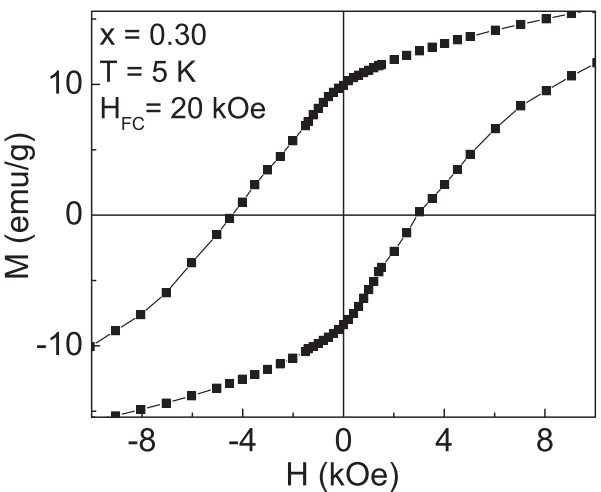

FIG. 7. Hysteresis loop taken at $5 \mathrm{~K}$ cooling from RT under a $20 \mathrm{kOe}$ magnetic field for the $\left(\mathrm{Fe}_{79} \mathrm{Mn}_{21}\right)_{70} \mathrm{Cu}_{30}$ alloy.

$f c c-\mathrm{FeMnCu}$ AFM phase but contribution from Pauli paramagnetism cannot be ruled out. ${ }^{20}$

Hysteresis loops were also recorded after field cooling $\left(H_{F C}=20 \mathrm{kOe}\right)$ the $\mathrm{x}=0.00,0.20$, and 0.30 samples from $300 \mathrm{~K}$. A shift to negative fields was observed, being this effect more evident at lower temperatures and for the highest $\mathrm{Cu}$ content $(\mathrm{x}=0.30)$, (see Fig. 7). To quantify this shift, we have estimated the exchange bias field $\left(\mathrm{H}_{\mathrm{EB}}\right)$ as ${ }^{21}$

$$
H_{E B}=\frac{\left|H_{C R}\right|-\left|H_{C L}\right|}{2},
$$

where $H_{C R}$ and $H_{C L}$ are the right and left coercive fields, respectively. The $H_{E B}$ absolute values are shown in Fig. 8, as a function of temperature for the three analyzed samples. We observe that, independently on the $\mathrm{Cu}$ concentration, $H_{\mathrm{EB}}$ is only appreciable below $T_{\mathrm{ZFC}}$, i.e., below the temperature of

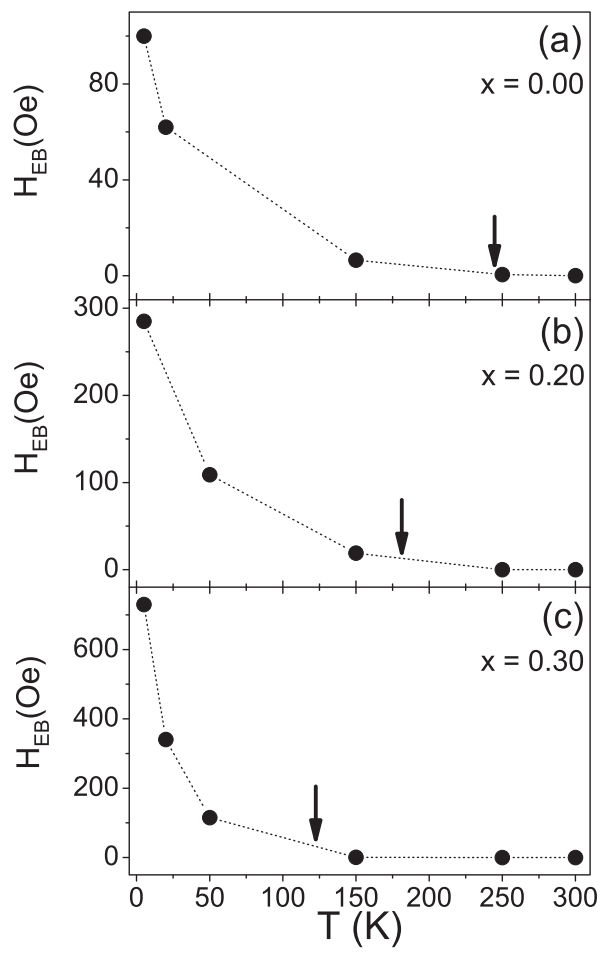

FIG. 8. Absolute value of the exchange bias field as a function of the temperature for $x=0.00$ (a), 0.20 (b), and 0.30 (c) samples. The arrows indicate the characteristic temperatures obtain from ZFC measurements. freezing of the moments belonging to the disordered regions. As the temperature decreases below $T_{\mathrm{ZFC}}, H_{E B}$ increases up to different values depending on the $\mathrm{Cu}$ content, i.e., the more $\mathrm{Cu}$ content implies the larger $H_{E B}$ (see Fig. 8).

Due to finite size effects the $f c c$ phase of the $\left(\mathrm{Fe}_{79} \mathrm{Mn}_{21}\right)_{1-\mathrm{x}} \mathrm{Cu}_{\mathrm{x}}$ nanostructures might present a net magnetic moment from uncompensated spins of the AFM sublattices. The local structural disorder due to distortions in coordination and bonds of those atoms at the intergrain zones leads to the formation of disordered regions. Both $T_{\mathrm{ZFC}}$ and $T_{\text {susc }}$ are related to the onset of the freezing into a SG-like state of the spin moments belonging to such regions. Furthermore, due to the complex nature of these nanostructures random magnetic interactions likely correlate the spin moments forming clusters that freeze collectively into a super-SG state. ${ }^{10}$ Within this uncompensated AFM/disordered region description, the moments at intergrain boundaries freeze below $T_{\mathrm{ZFC}}$ and align with the uncompensated ones. Thus, analogously to the case of the EB effect observed in oxide nanoparticles, ${ }^{22,23}$ here the EB might arise from coupling interactions at the interface between the AFM phases and the spin-glass-like intergrain boundaries.

The loop shift effect under field cooling was analyzed in more detail for the $\mathrm{x}=0.30$ sample, which is the sample that presents a more apparent glassy behavior. To this end, $H_{\mathrm{EB}}$ values were estimated from different cooling procedures performed on this alloy (Figs. 9(a) and 9(b)). On one hand,
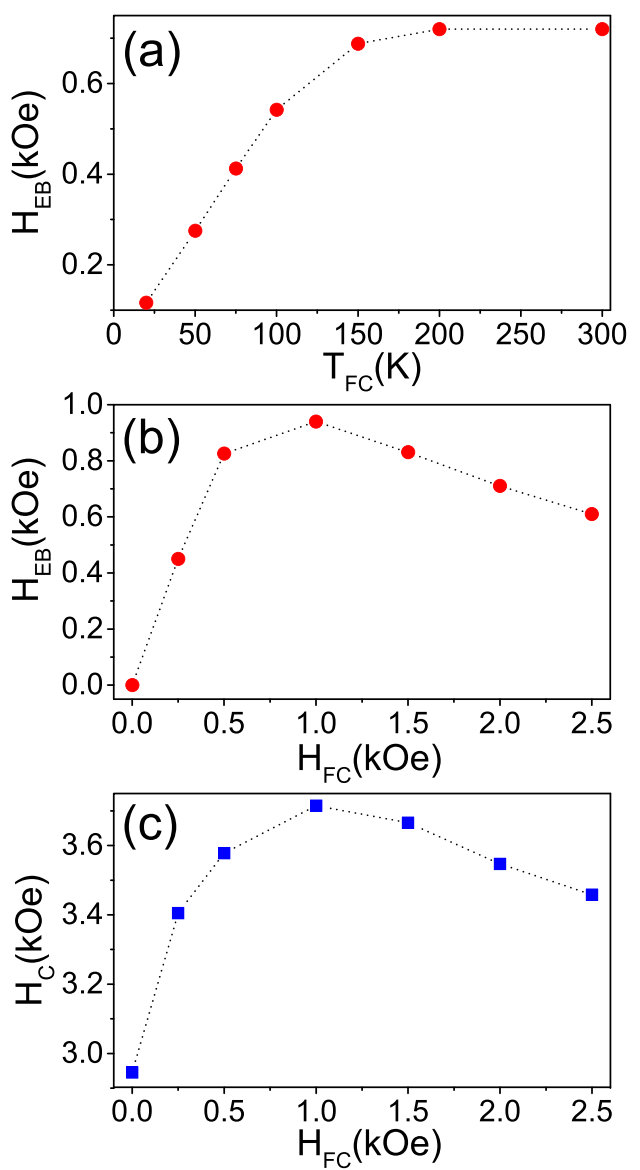

FIG. 9. (a) Exchange bias field corresponding to a field cooling of $20 \mathrm{kOe}$ applied from $T_{H F C}$ down to $5 \mathrm{~K}$ to the $\mathrm{x}=0.30$ sample. (b) Exchange bias and (c) coercive field, as a function of the applied cooling magnetic field for the $\mathrm{x}=0.30$ sample registered at $5 \mathrm{~K}$. 
$H_{F C}=20 \mathrm{kOe}$ is applied from a variable temperature $\left(T_{H \mathrm{FC}}\right)$ until $5 \mathrm{~K}$; at this point the hysteresis loop measurement is performed. These results show that when $H_{\mathrm{FC}}$ is applied from temperatures $T_{H \mathrm{FC}}>T_{\mathrm{ZFC}}=120 \mathrm{~K}$, the loop shift is quite significant and $\mathrm{H}_{\mathrm{EB}}$ remains almost unalterable. However, when $T_{H \mathrm{FC}}<T_{\mathrm{ZFC}}, H_{E B}$ decreases as $T_{H \mathrm{FC}}$ diminishes (Fig. 9(a)). On the other hand, field cooling cycles were measured from 300 down to $5 \mathrm{~K}$ under cooling fields $H_{F C}$ from 0 Oe to $2.5 \mathrm{kOe}$. We observe that the hysteresis-loop shift is more pronounced as $H_{F C}$ increases, reaching a maximum $H_{E B}$ absolute value of $940 \mathrm{Oe}$ when $H_{F C}=1.0 \mathrm{kOe}$ (see Fig. 9(b)) and afterwards it decreases gradually for higher $H_{\mathrm{FC}}$. Also, the coercive field shows an identical trend, i.e., it increases up to $1 \mathrm{kOe}$ and then decreases when increasing $H_{F C}$ (Fig. 9(c)).

Field-cooling dependence of $H_{\mathrm{EB}}$ has been reported to occur in Fe nanoparticles (FM phase) embedded in an iron oxide matrix (disordered phase). ${ }^{21}$ Such behavior was attributed to the interfacial exchange coupling between these two coexisting magnetic phases, being the spin configuration of the disordered phase influenced by the cooling field. On the other side, numerical results demonstrated that the cooling field affects the hysteresis behavior of core-shell structures with a SG-like shell. ${ }^{24,25}$ Similarly, in our case, by applying $H_{F C}$ at temperatures higher than the freezing temperature $(120 \mathrm{~K})$ for $\left(\mathrm{Fe}_{79} \mathrm{Mn}_{21}\right)_{0.70} \mathrm{Cu}_{0.30}$ establishes a preferential alignment direction for the magnetic moments at the interfaces. ${ }^{21}$ Field-cooling the sample from $T>120 \mathrm{~K}$ freezes the moments of intergranular regions in a disordered state determined by the competing energy terms: exchange interactions between spins at the AFM/SG-like interfaces and the Zeeman energy. When cooling fields are less than $1.0 \mathrm{kOe}$, the exchange anisotropy dominates below the freezing temperature and a shifted loop is observed. Under cooling fields higher than $1.0 \mathrm{kOe}$ the moments tend to align along the field direction and the Zeeman energy overcomes the exchange anisotropy energy and $H_{E B}$ decreases. In the presence of a magnetic field, the spin configuration relax to find its lowest energy state. Field cooling at different temperatures causes the loop shift depends on the initial spin configurations.

\section{CONCLUSIONS}

We have presented a systematic study of the magnetic properties of alloys $\left(\mathrm{Fe}_{79} \mathrm{Mn}_{21}\right)_{1-\mathrm{x}} \mathrm{Cu}_{\mathrm{x}}(0.00 \leq \mathrm{x} \leq 0.30)$. This system is a multi-phase system from a magnetic point of view. The main phase is antiferromagnetic with structurally disordered regions (intergrain boundaries) with spin glass-like magnetic behavior. The alloys show displaced hysteresis loops when cooling down under an applied field from above the freezing temperature of the disordered regions. This is originated in the exchange anisotropy at the AFM/SG-like interfaces. When the applied magnetic fields are higher than $4 \mathrm{kOe}$ the magnetic anisotropy disappears, i.e., such a field is high enough to overcome the disordered spin configuration driven by the magnetic frustration present in the disordered phase. These results open an interesting perspective for manufacturing new metallic materials with $\mathrm{AFM}$ /disordered region with exchange bias behavior driven by the applied field.

\section{ACKNOWLEDGMENTS}

This work is dedicated to the memory of Professor Judith Desimoni, who passed away when these results were under a discussion stage. Research Grant PIP 0230 from CONICET, Argentina, and BID 1728 OC/AR PICT 38047 from the ANPCyT are gratefully recognized. The DC magnetic measurements were performed using the RN3M facilities.

${ }^{1}$ J. Nogués, J. Sort, V. Langlais, V. Skurmryev, S. Suriñach, J. S. Munoz, and M. Baró, Phys. Rep. 422, 65 (2005).

${ }^{2}$ R. L. Comstock, J. Mater. Sci.: Mater. Electron. 13, 509 (2002).

${ }^{3}$ S. Giri, M. Patra, and S. Majumdar, J. Phys.: Condens. Matter 23, 073201 (2011).

${ }^{4}$ W. H. Meiklejohn and C. P. Bean, Phys. Rev. 102, 1413 (1956).

${ }^{5}$ A. Punnoose, H. Magnone, M. S. Sheehra, and J. Bonevich, Phys. Rev. B 64, 174420 (2001).

${ }^{6}$ R. D. Zysler, E. Winkler, M. V. Mansilla, and D. Fiorani, Physica B 384, 277 (2006).

${ }^{7}$ A. Hernando, E. Navarro, M. Multigner, A. R. Yavari, D. Fiorani, M. Rosenberg, G. Filoti, and R. Caciuffo, Phys. Rev. B 58, 5181 (1998).

${ }^{8}$ F. Jiménez-Villacorta, J. L. Marion, T. Sepehrifar, and L. H. Lewis, J. Appl. Phys. 111, 07E141 (2012).

${ }^{9}$ A. E. Bianchi, S. J. Stewart, R. D. Zysler, and G. Punte, J. Appl. Phys. 112, 83904 (2012).

${ }^{10}$ J. A. De Toro, M. A. Lopez de la Torre, J. M. Riveiro, J. Bland, J. P. Goff, and M. F. Thomas, Phys. Rev. B 64, 224421 (2001).

${ }^{11}$ M. Mizrahi, A. F. Cabrera, S. J. Stewart, H. E. Troiani, S. M. Cotes, and J. Desimoni, Physica B 354(1), 133 (2004).

${ }^{12}$ M. Mizrahi, A. F. Cabrera, and J. Desimoni, J. Appl. Phys. 107(4), 044311 (2010).

${ }^{13}$ Q. Zhang, D. Li, W. B. Cui, J. Li, and Z. D. Zhang, J. Appl. Phys. 106, 113915 (2009).

${ }^{14}$ J. A. De Toro, M. A. López de la Torre, M. A. Arranz, J. M. Riveiro, and J. L. Martínez, Phys. Rev. B 64, 094438 (2001).

${ }^{15}$ J. A. Mydosh, Spin Glasses: An Experimental Introduction (Taylor \& Francis, London-Washington, DC, 1993).

${ }^{16}$ J. L. Dormann, L. Bessais, and D. Fiorani, J. Phys. C: Solid State Phys. 21, 2015 (1988)

${ }^{17}$ S. Shtrikman and E. P. Wohlfarth, Phys. Lett. A 85(8-9), 467 (1981).

${ }^{18}$ J. R. L. de Almeida and D. J. Thouless, J. Phys. A 11, 983 (1978).

${ }^{19}$ M. B. Stearns and Y. D. Cheng, J. Appl. Phys. 75(10), 6894 (1994).

${ }^{20} \mathrm{~S}$. Blundell, Magnetism in Condensed Matter, 2nd ed. (Oxford University Press, 2003), p. 143.

${ }^{21}$ L. Del Bianco, D. Fiorani, A. M. Testa, E. Bonetti, and L. Signorini, Phys. Rev. B 70, 052401 (2004).

${ }^{22}$ M. Gruyters, Phys. Rev. Lett. 95, 077204 (2005).

${ }^{23}$ M. Ali, P. Adie, C. H. Marrows, D. Greig, B. J. Hickey, and R. L. Stamps, Nature Mater. 6, 70 (2007).

${ }^{24}$ M. Vasilakaki and K. N. Trohidou, Phys. Rev. B 79, 144402 (2009).

${ }^{25}$ O. Iglesias, X. Batlle, and A. Labarta, J. Phys. D: Appl. Phys. 41, 134010 (2008). 